\title{
REPRESENTACIONES SOCIALES SOBRE LA RECREACIÓN, EL DEPORTE Y LA EDUCACIÓN FÍSICA DE LOS PROFESORES ESPECIALISTAS DEL PROGRAMA DE LICENCIATURA EN EDUCACIÓN BÁSICA CON ÉNFASIS EN EDUCACIÓN FÍSICA, RECREACIÓN Y DEPORTES DE LA UNIVERSIDAD DE PAMPLONA.
}

Contreras. Dennis ${ }^{1}$, Orjuela. Ailin², Peñaloza. Sergio³

\section{Doctor en Educación, Profesor Universidad de Pamplona. Colombia}

2. Magister en Ciencias Computacionales. Profesora Universidad de Pamplona, Colombia.

3. Maestrante en Gestión de Proyectos Informáticos. Universidad de Pamplona, Colombia.

\section{RESUMEN}

El propósito de este estudio fue analizar la configuración de las representaciones sociales de los docentes del área sobre la educación física, el deporte y la recreación, del programa de licenciatura en educación básica con énfasis en educación física, recreación y deportes de la Universidad de Pamplona. El enfoque epistemológico planteado para el desarrollo de esta investigación corresponde al pragmatismo, el diseño fue el secuencial exploratorio y el método fue la teoría fundamentada. El procedimiento de la recopilación de datos e información se realizó en dos fases: Fase Cualitativa y Fase Cuantitativa. En la fase cualitativa se llevó a cabo la entrevista a profundidad, se realizaron 6 entrevistas (dos a cada docente participante de la investigación, con una diferencia entre cada una de ella de ocho (8) días), en la fase cuantitativa se llevó a cabo la encuesta y se realizaron cuarenta y cinco encuestas. Todos los documentos que resultaron de la información recopilada en la fase cualitativa fueron exportados y analizados en el programa Atlas.ti., la fase cuantitativa fue analizada por medio de un análisis de correspondencia.

Palabras clave: Educación, Educación física, epistemología, sociedad.

\begin{abstract}
The purpose of this study was to analyze the configuration of social representations of teachers in the area of physical education, sport and recreation of the undergraduate program in basic education with emphasis on physical education, recreation and sports of the University of Pamplona. The procedure of the collection of data and information is performed in two phases: phase qualitative and phase quantitative. In the qualitative phase was conducted the interview in depth, 6 interviews were conducted (two each teacher participant research, with a difference between each one of eight (8) days). in the phase quantitative is led to out the survey and was performed forty and five surveys. All the documents that resulted from the information collected in the qualitative phase were exported and analyzed in the program Atlas.ti., the quantitative phase was analyzed by means of a correspondence analysis.
\end{abstract}

Keyword: Education, physical education, epistemology, society. 


\section{INTRODUCCIÓN}

La representación social en una modalidad particular de conocimiento, cuya función es la elaboración de los comportamientos y la comunicación de los individuos. La representación es un corpus organizado de conocimientos y de las actividades psíquicas gracias a las cuales los hombres hacen intangible la realidad física y social, se integra en un grupo o una relación cotidiana de intercambios, libera los poderes de su imaginación (Moscovici, 1979; citado por Mora, 2002).

El término hace referencia tanto al proceso como al producto de la construcción mental de la realidad. Por otra parte, las representaciones condensan y sintetizan características del objeto representado y del sujeto que lo representa. Funcionalmente, las representaciones sociales clasifican a los objetos sociales, los explican y evalúan sus características a partir del discurso y las creencias de sentido común ((D'Andrea, A.M. y otros, 2006).

Uno de los lugares más críticos en los que se procesan estas ideas es en las diferentes instituciones de formación docente, debido a que es allí donde se certifican las competencias necesarias de quien participará en la producción del sujeto acorde con el orden propuesto por el sistema social (D'Andrea, A.M. y otros, 2006). El sistema educativo fabrica juicios y jerarquías de excelencia que a menudo recrean otras formas de calificación, incluso informales. Los juicios y jerarquías de excelencia son, en tanto representaciones, el resultado de la construcción intelectual, cultural y social (D'Andrea, A.M. y otros, 2006).
En toda organización existen representaciones compartidas acerca de aquellos objetos, hechos, conductas, etc., que la misma valora y considera importantes. En esta perspectiva se ubica esta investigación, con el propósito de presentar la descripción de los contenidos de las representaciones sociales de los profesores, referente a las representaciones sociales sobre la recreación, el deporte y la educación física. El departamento de educación física de la Universidad de Pamplona se considera de interés para el estudio porque en él los futuros docentes se inician en la teoría y en la práctica valorativa y evaluativa en todas sus dimensiones.

Las finalidades de esta área se centran en el desarrollo humano y social, en un proyecto educativo que privilegia la dignidad humana, la calidad de vida, el desarrollo de la cultura y el conocimiento, la capacidad de acción y la participación democrática. Se reconoce la educación física, recreación y deportes como una práctica social del cultivo de la persona como totalidad en todas sus dimensiones (cognitiva, comunicativa, ética, estética, corporal, lúdica), y no sólo en una de ellas. El reto inicial de los lineamientos curriculares es cómo pensar, hacer y enseñar una educación física que dé respuesta a los factores que intervienen en su realización, en donde se relacionan e interactúan el saber disciplinar, el contexto sociocultural y el sujeto, a través de prácticas pedagógicas orientadas a la formación personal y social afirmada en una reflexión sobre lo que ha sido, un análisis de la situación actual y una exploración de perspectivas y compromisos hacia el deber ser de esta área educativa fundamental. 
Lo anteriormente descrito incide en el concepto de cada uno de los actores que interviene en el proceso pedagógico de la educación física, y produce una situación compleja, en el cual se cruzan múltiples componentes y maneras de relación. Por lo cual se necesita una visión crítica y objetiva, aspectos claves que oriente su construcción en los diferentes campos. Por tanto el estudio de las representaciones sociales de los profesores está mereciendo la atención de organizaciones profesionales ocupadas en la tesis de desvelar los procesos que gobiernan las acciones de los profesores. En el fondo del contexto del análisis se encuentra la preocupación de preparar a los profesores conociendo más aspectos de las razones que les inducen a obrar como lo hacen.

\section{Marco Metodológico}

\section{Enfoque Epistemológico}

El enfoque epistemológico planteado para el desarrollo de esta investigación corresponde al pragmatismo, dado que este enfoque reconoce que el valor del conocimiento depende de los métodos con los cuales se obtuvo, es así que el estudio combina métodos de la investigación cualitativa para tratar de interpretar las Representaciones Sociales sobre la Recreación, el Deporte y la Educación Física de los profesores especialistas en el área. Caso de estudio: Universidad de Pamplona, en este caso los resultados del primer método cualitativo permiten ayudar a desarrollar o informar el segundo método cuantitativo (Greene \& Caracelli, 2003). El pragmatismo como enfoque epistemológico privilegia el énfasis práctico sobre la investigación lo que es muy pertinente para poder analizar las representaciones sociales sobre la recreación, el deporte y la educación física.

\section{Diseño Mixto}

Para realizar esta investigación se utilizó el Diseño Secuencial Exploratorio, presentado por (Tashakkori y Teddlie, 2003; citado por Mora, 2002). La investigación fue exploratoria, aplicada desde los métodos cualitativo y cuantitativo de forma secuencial con la intención de Interpretar las relaciones dadas entre las representaciones sociales del área, su práctica pedagógica, el currículum y su ejecución en el programa de licenciatura en educación con énfasis en educación física, recreación y deportes de la Universidad de Pamplona. En este caso los resultados del primer método cualitativo permiten ayudar a desarrollar 0 informar el segundo método cuantitativo (Balcells, 2013). La condición básica de este diseño radica en que se precisa la exploración previa, ya que no se dispone de instrumentos 0 medidas, se desconocen las variables, y tampoco existe marco teórico.

Este diseño se inicia con la obtención de datos cualitativos para explorar el fenómeno, construyéndose después una etapa cuantitativa, y cuyos resultados se conectarán con la fase cualitativa. Este es un diseño de investigación en el cual, a través del proceso de un estudio, se aplican fundamentos cualitativos $y$ cuantitativos. La integración de los fundamentos puede darse desde la selección de marcos filosóficos, paradigmáticos y teóricos; la construcción de las preguntas; el desarrollo de la metodología; la selección de métodos; el proceso de colección de datos; los procedimientos de análisis; y el desarrollo de inferencias (Creswell, Clark, Gutmann, \& Hanson, 2003; Creswell \& Plano Clark, 2007, 2011; Greene \& Caracelli, 2003; Hesse-Biber, 2010; Tashakkori \& Teddlie, 2003a, 2010). Para fines de esta investigación integro los criterios metodológicos principalmente de Creswell, Tashakkori y Teddlie. 
El diseño de esta investigación consiste en preguntas de tipo exploratorias; la recopilación de datos cualitativos y cuantitativos de forma secuencial con el último dependiente del primero, priorizando en el método cualitativo, analizados de forma separada y la formulación de inferencias. Además se presentó una metainferencia a partir de las inferencias que surgieron de ambas fases.

En resumen, esta investigación asume el enfoque epistemológico pragmático desde un diseño con método mixto denominado Exploratorio Secuencial (Creswell, 2009) el cual se caracteriza por una implementación en dos fases, de allí lo secuencial: una cualitativa que recoge datos y analiza; otra cuantitativa que también recoge datos y analiza. Ambas fases e integran en la teorización pues allí se utilizan los encontrado en la segunda para corroborar la interpretación cualitativa. Este tipo de diseño lo que pretende es potenciar los hallazgos desde ambos métodos en un solo estudio.

\section{Método: Teoría Fundamentada}

La teoría fundamentada es una estrategia metodológica que tiene como propósito, final generar 0 descubrir una teoría. Su objetivo es la construcción de un esquema de análisis con altos niveles de abstracción sobre un fenómeno social específico (Sandín 2003).

Los procedimientos para desarrollar la teoría fundamentada propuesta por Glaser y Straus (1967), se centran en el método de la comparación constante y el muestreo teórico, estos procedimientos se conforman como las contribuciones metodológicas básicas de esta teoría, en el desarrollo de los procesos de investigación social.

El Método de Comparación Constante propone realizar simultáneamente procesos de codificación y análisis, con el propósito de ir generando la teoría de forma sistemática, por medio de la utilización de la codificación explícita y de procedimientos analíticos. Se trata entonces de ir paralelamente combinando en el análisis de los datos cualitativos, conceptos, categorías, propiedades, hipótesis e hipótesis interrelacionadas, teniendo presente la posibilidad del surgimiento de nuevas categorías con sus propiedades, las cuales deben ser incorporadas en el proceso de análisis. Una ventaja de este método es el hecho de que facilita al investigador la generación de teoría con características de: "... integrada, consistente, plausible y cercana a los datos". (Kornblit 2004:50, citado por Hernández, 2011).

La aplicación de este método no sólo abre la posibilidad para generar una teoría que esté en línea directa de correspondencia con los datos, sino que además, permite al investigador examinar una gran variedad de datos. En otras palabras, cada uno de los incidentes debe ser comparado con otro o comparado con las propiedades de una categoría.

\section{Técnicas de Recolección Datos: Entrevista en Profundidad y Encuesta}

\section{Entrevista a Profundidad.}

La entrevista en profundidad 0 , como algunos investigadores/as la denominan, el estudio de caso (Robert, 1994), es la entrevista profesional que se realiza entre un entrevistador/a y un informante con el objeto de obtener información sobre la vida, en general, 0 sobre un tema, proceso o experiencia concreta de una persona. A través de la entrevista en profundidad, el entrevistador/a quiere conocer lo que es importante y significativo para el entrevistado/a; llegar a comprender como ve, clasifica e interpreta su mundo en general 0 algún ámbito o tema que interesa para la investigación, en particular (Seltiz, 1980). 


\section{El Cuestionario}

Para Ander-Egg (2000), el cuestionario contiene "aspectos del fenómeno que se consideran esenciales, permite además aislar ciertos problemas que interesan, principalmente reduce la realidad a cierto número de datos esenciales y precisa el objeto de estudio." A través de éste se obtuvo los datos cuantitativos de la investigación.

\section{Proceso de reclutamiento de las personas participantes para las entrevistas.}

Se proyectó que las entrevistas participarían docentes del departamento de educación física recreación y deportes con las siguientes características:

- Como mínimo 6 años sin interrupción como docentes del departamento de educación física recreación y deportes.

- Tener grado de maestría en el área de perfil de las asignaturas asignadas como responsabilidad académica.

- Ser docente de pregrado y postgrado, como mínimo 3 años de experiencia en la cátedra de postgrado.

- Tener un promedio de evaluación semestral como docentes no inferior a 4.5 .

\section{Consideraciones Éticas}

Uno de los aspectos indispensables en los procesos de investigaciones humanas $y$ sociales, y para la profesión de trabajo social en general, es el asunto de la ética. Este investigación cumplió con los estándares para la realización de investigaciones en seres humanos, según las disposiciones de la Convención de Helsinki (1968) y las normas científicas, técnicas y administrativas para la investigación en salud, según resolución número 8430 de 1993 del Ministerio de Salud. Colombia.
Se les entrego cartas de presentación de la investigación y hojas de consentimiento y asentimiento informado a las docentes participantes. En estos documentos se explica el propósito y los objetivos de la investigación; las garantías de privacidad, confidencialidad y anonimato; sus derechos; los riesgos $y$ beneficios. Además, se especificó, en las comunicaciones, que este estudio no conllevaba riesgos significativos a la persona participante $\mathrm{ni}$ a la institución que dicha persona representa 0 asiste. Además, se les informó de modo verbal y por escrito que este proceso era voluntario y que se podían retirar en cualquier momento de la investigación, sin penalización alguna.

En el caso de los cuestionarios de la encuesta, se identificó a cada participante con un número de control garantizando así su anonimato de cada participante, así mismo las hojas de datos sociodemográficos. Se cumplió, a través de todo el proceso de la investigación, con la ética de respeto a la subjetividad, particularidad y relatividad, sin negar la relevancia en principios universales como la justicia y la igualdad. Cumpliendo con el derecho que tienen las personas participantes de conocer los resultados de la investigación.

\section{Etapas de recolección de datos}

El escenario donde se desarrolló la investigación fue la Universidad de Pamplona, Pamplona Norte de Santander, Colombia, Facultad de Salud, Departamento de Educación de Física Recreación y Deportes, de enero a junio de 2014. Se seleccionó el departamento de educación física recreación y deportes considerando sus cuarenta años formado profesionales en el área de la educación física, el talento humano (docentes del departamento), su accesibilidad y la disponibilidad. Al momento de la investigación el departamento de educación física recreación y deportes contaba 
con treinta (30) docentes (9 mujeres y 21 hombres).

El procedimiento de la recopilación de datos e información se realizó en dos fases: Fase Cualitativa y Fase Cuantitativa. En la fase cualitativa se llevó a cabo la entrevista a profundidad, se realizaron 6 entrevistas (dos a cada docente participante de la investigación, con una diferencia entre cada una de ella de ocho (8) días), en la fase cuantitativa se llevó a cabo la encuesta y se realizaron cuarenta y cinco encuestas. Todos los documentos que resultaron de la información recopilada en la fase cualitativa fueron exportados y analizados en el programa Atlas.ti., la fase cuantitativa fue analizada por medio de un análisis de correspondencia.

\section{Fase I Cualitativa \\ Proceso de recopilación de información de las entrevistas}

Las entrevista se realizaron el departamento de educación física recreación y deportes de la Universidad de Pamplona, previamente se había firmado un consentimiento informado por la dirección de la facultad, del departamento y de cada uno de los docentes. La duración de las entrevistas fue de media hora.

\section{Descripción de la guía de entrevistas}

El guion se organizó de la siguiente manera: (a) los significados de conceptos relacionados con educación física, recreación, actividad física y deporte; (b) los perfiles relacionados con la responsabilidad académica, (c) el plan de estudio 2006; (d) necesidades nacional e internacional del currículo. Se desarrolló, además, una hoja de datos sociodemográficos para los docentes entrevistados En ésta, la persona informante indicó la edad, el sexo, la nacionalidad, la preparación académica, el tiempo que ejerció la profesión, los años de servicio en el sistema de educación pública, el puesto que ocupa al momento, el tiempo que llevaba ocupándolo y la responsabilidad o el cargo oficial adicional que tiene en el departamento de educación física recreación y deportes.

Proceso de análisis de las Entrevistas con el Atlas Ti.

El análisis de contenido es una técnica de investigación destinada a formular a partir de ciertos datos, inferencias reproducibles y válidas que pueden aplicarse a su contexto. De esta manera es una técnica apropiada para la descripción objetiva sistemática y cualitativa del contenido manifiesto o implícito de una fuente de datos como las entrevistas. El investigador cualitativo que hace uso del análisis de contenido asume la responsabilidad de interpretar lo que se observa, escucha o lee (Krippendorff, 1990).

Siendo que el procedimiento descrito reviste complejidad surge la recomendación metodológica, usar el programa computacional Atlas.ti como herramienta de apoyo al proceso de análisis. Su fundamentación teórica se basa en la Teoría Fundamentada (Grounded Theory) de Glaser y Strauss (1967), su aplicación está llevando a los más diversos campos de las humanidades: psicología, sociología, antropología, educación, economía, ciencia política, etc.

En el programa Atlas.ti, el proceso implica cuatro etapas: Codificación de la información (de los datos); Categorización; Estructuración o creación de una o más redes de relaciones 0 diagramas de flujo, mapas mentales o mapas conceptuales, entre las categorías; y Estructuración de hallazgos o teorización si fuere el caso. El Atlas.ti ofrece 6 tipos de relación y su símbolo, pero el investigador puede crear muchos otros con sus posibles símbolos, de acuerdo a sus necesidades y conveniencias. En la tabla 1, se ejemplifican algunos de los más importantes. 
Link to code: Contagio

$==\quad$ : is associated with

$\square \quad$ : is part of

$=>\quad$ : is cause of

$<>$ : contradicts

isa : is a

: noname

$\Rightarrow$ : is property of

Open Relation Editor

Tabla 1. Tipos de Relaciones entre Códigos.

La aplicación del análisis de contenido como del ATLAS.ti implican el cumplimiento de procedimientos pero la selección de las unidades de análisis tiene un significado de importancia que lo decide el investigador. La asignación, definición y relación de códigos tiene una carga interpretativa que es del investigador. $Y$ la denominación (nombre de la categoría) y definición de las categorías son ideas propias del investigador. Lo que indica que el análisis cualitativo de la información y el uso del programa computacional ATLAS.ti como herramienta de apoyo deben relacionarse por la creatividad del investigador (a) enriqueciéndose así los resultados del análisis.

\section{Fase II Cuantitativa}

\section{Encuesta. Análisis de Correspondencias}

En esta fase se utilizó la encuesta como técnica de recopilación de datos cuantitativos, la cual provee una descripción cuantitativa de opiniones, creencias, actitudes y prácticas sobre las representaciones sociales del área, su práctica pedagógica, el currículum y su ejecución en el programa de educación física, el deporte y la recreación de la Universidad de Pamplona. Esta estrategia de recopilación de datos facilita la administración a grupos y a muestras por disponibilidad. Además, el por ciento de respuesta es alto debido a que la percepción de anonimato es mayor, y la validez es de alta a moderada en cuestionarios bien construidos y pilotados (Johnson \& Turner, 2003).

Su versión definitiva se realizó a previa consulta a expertos y la realización de un ensayo piloto que permitió poner a prueba el instrumento, reformular de nuevo algunos ítems y eliminar aquéllos poco discriminativos (Azorín y Sánchez Crespo, 1986). La construcción de los ítems se ajusta en su mayoría a la modalidad escalar de Likert. En el cuestionario se han considerado dos grandes bloques, que pueden visualizar el las dos siguientes tablas:

\begin{tabular}{|l|l|l|}
\hline $\begin{array}{c}\text { Dimensión } \\
\text { Profesional }\end{array}$ & \multicolumn{1}{|c|}{ Aspectos Estudiados } & $\begin{array}{l}\text { Proposiciones de } \\
\text { Cuestionario }\end{array}$ \\
\hline & $\begin{array}{l}\text { 1. Edad, } \\
\text { 2. Sexo. }\end{array}$ & A, B \\
\hline Identificación & & \\
\hline & $\begin{array}{l}\text { 1. Modelos profesionales } \\
\text { 2. Percepción de la formación inicial }\end{array}$ & $37,38,39,40,41$, \\
3ercepciones & $\begin{array}{l}\text { 3. Percepción de la incidencia de } \\
\text { factores sociales y personales } \\
\text { sobre el rendimiento escolar. } \\
\text { Profesionales }\end{array}$ & \\
& 4. Percepción de asignatura & \\
\hline
\end{tabular}

Tabla 2. Distribución de las proposiciones del cuestionario para la dimensión profesional.

\begin{tabular}{|c|c|c|}
\hline $\begin{array}{l}\text { Dimensión } \\
\text { Profesional }\end{array}$ & Aspectos Estudiados & $\begin{array}{c}\text { Proposiciones del } \\
\text { Cuestionario }\end{array}$ \\
\hline Contenidos & $\begin{array}{l}\text { 1. Relación con otros conocimientos } \\
\text { 2. Fuentes y organización }\end{array}$ & $\begin{array}{l}28,29,30,31,32 \\
33,34,35,36\end{array}$ \\
\hline Evaluación & $\begin{array}{l}\text { 1. Instrumentos } \\
\text { 2. Diseño y organización } \\
\text { 3. Finalidad }\end{array}$ & $\begin{array}{l}1,2,3,4,5,6,7,8 \\
9,10,11\end{array}$ \\
\hline Metodología & $\begin{array}{l}\text { 1. Planificación } \\
\text { 2. Desarrollo de la enseñanza } \\
\text { 3. Adaptación al alumno } \\
\text { 4. Motivación y participación } \\
\text { 5. Recursos }\end{array}$ & $\begin{array}{lllll}12, & 13, & 14, & 15, & 16 \\
17, & 18, & 19, & 20, & 21 \\
22, & 23, & 24, & 25 & 26 \\
27 & \end{array}$ \\
\hline
\end{tabular}

Tabla 3. Distribución de las proposiciones del cuestionario para las dimensiones curriculares.

Se realizó un análisis de correspondencias, que es un método de análisis de datos que representa gráficamente tablas de datos. El 
análisis de correspondencias es una generalización de una representación gráfica denominada diagrama de dispersión. Para el análisis de correspondencias, es conveniente reflexionar sobre lo que se entiende por diagrama de dispersión y sobre cómo se interpreta los datos que éste representa gráficamente.

\section{Proceso de Análisis e Interpretación}

El proceso de análisis e interpretación de la información recopilada se estructuró a partir del diseño mixto secuencial exploratorio (DMSE). Según el diseño aplicado, analicé los datos cualitativos y la información cuantitativa de modo secuencial, en ese orden. Primero, analicé e interpreté los datos obtenidos de las entrevistas (Fase cualitativa) y, seguidamente, la información obtenida de la encuesta (Fase cuantitativa). Paso a explicar los tipos de análisis de ambos métodos, luego explico las triangulaciones realizadas.

\section{RESULTADOS}

\section{Fase I Cualitativa.}

A continuación se presentan la primera fase del análisis cualitativo, en tanto descripción del fenómeno, como su respectivo análisis e interpretación de los mismos. Los datos cualitativos seleccionados son básicamente discurso, hechos de lenguaje, ya que poseen un significado más allá de su explícita enunciación. Ese lenguaje es fundamentalmente simbólico, lo que lo convierte en la materia prima de las representaciones sociales.

Las entrevistas fueron realizadas a lo largo del estudio. Seis (6) docentes participaron, voluntariamente, en las entrevistas, grabadas en audio digital y posteriormente transcritas en su totalidad. Para categorizar las afirmaciones que juzgamos relevantes para el trabajo, se optó por utilizar el tema como unidad de análisis. Con base en criterios esencialmente semánticos, creamos, a posteriori, unidades temáticas y sus respectivas categorias, donde se agruparon las afirmaciones que expresaban, explícita 0 implícitamente, creencias, opiniones, y sentidos sobre el objeto de estudio investigado (Strauss y Corbin, 2002).

Las afirmaciones fueron organizadas, donde, se refiere a una unidad temática elaborada. Los entrevistados son identificados por su dos primeras letras del nombre ficticio, seguidamente por un número relacionado a orden de realización de las entrevistas y su respectiva titulación académica ( $\mathrm{M}$ : magister y D: doctor).

Este primer análisis de los datos ha permitido realizar una representación basada en las relaciones entre la categoría inicial y las dimensiones que la conforman, basada en la codificación axial de Strauss y Corbin (2002). Esto permitió agruparlas como un primer nivel para relacionar la categoría, sus dimensiones y propiedades. A continuación se presenta un diagrama de las relaciones establecidas entre dicha categoría y sus dimensiones.

\section{Énfasis en Educación Física (Networks 1)}

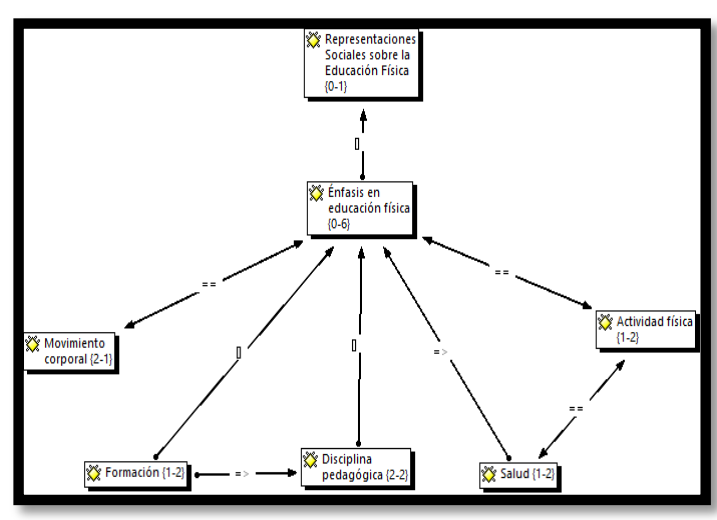

Figura 1. Énfasis en Educación Física 
La representación social en los informantes claves está conformada por un conocimiento cognitivo y de experiencia, ambos en el mismo plano de importancia aunque se evidencia hasta este punto un mayor peso en lo conceptual, lográndose la saturación teórica (Martínez, 1999; Strauss y Corbin, 2002,). Esto permite afirmar el valor explicativo de las dimensiones para precisar los elementos constitutivos de la categoría representación social de educación física. El diagrama representa esa relación de asociación entre conocimiento cognitivo y de experiencia, pues ambos son "parte" de la representación social de educación física.

La Educación Física siendo una disciplina que aborda al ser humano en sus diferentes dimensiones; debe ser asumida como una necesidad personal y colectiva que permita enriquecer la cultura y la convivencia a través de la implementación de herramientas que le lleven a intervenir de una manera adecuada en la formación de niños/niñas, jóvenes, capaces de ser agentes reguladores en su interacción con la realidad actual del país.

El cambio de valoración que lo corporal y el movimiento han experimentado como consecuencia de un complejo cúmulo de circunstancias de diverso orden, cultural, económico, ideológico, sociológico, etc., imponen una ineludible reflexión que devele la ampliación que el significado de estos conceptos están adquiriendo en la sociedad occidental. Una nueva comprensión del movimiento, la corporeidad y, desde ella, su papel en el proyecto docente permitirá ubicar la Educación Física, de manera eficaz, dentro de un proyecto global y coherente, donde se le considere como una actividad del hombre en toda su vida y en su totalidad. Por cuanto la exploración de la expresión corporal le ofrece al Licenciado de Educación Física la posibilidad de proponer un itinerario madurativo que va del placer de actuar al placer de pensar, proceso que se da de manera dialéctica, donde los diferentes componentes del proceso de enseñanza - aprendizaje interactúan permanentemente.

Dentro del mundo de la educación es común pensar que la Educación Física se limita a la práctica de ejercicios físicos, juegos y deportes sin mayor trascendencia en el ámbito intelectual y emocional obviando su relación con el desarrollo integral del individuo.

La Educación Física en más de un siglo de presencia en la escuela y la sociedad, ha construido imaginarios, prácticas, conceptos, organizaciones, modelos de enseñanza y aprendizaje que hoy se transforman en un contexto de globalización, diversidad, desarrollo tecnológico y tradición; de nuevas sensibilidades; búsqueda de libertad individual y cultura democrática que influencian la perspectiva curricular hacia la atención de nuevas competencias humanas y apertura a una nueva visión del ser humano y de la nación.

Desde una perspectiva ampliada la Educación Física es una disciplina que se ocupa del ser humano, buscando la integridad, el equilibrio físico, emocional, intelectual y social. Con el propósito de identificar habilidades que sirvan de base para alcanzar destrezas en otros campos de desarrollo y acceder a una vida productiva en los ámbitos de la autonomía personal, de la familia y sobre el medio ambiente; respondiendo a dicha realidad el Licenciado de Educación Física no solo debe ver y estudiar la Educación física formal sino todos los otros aspectos que el hombre hace y vive. No se pretende focalizar en una etapa de la vida del hombre o en un solo aspecto de su movimiento. Se requiere un Licenciado que 
pueda orientar al hombre en cualquier nivel y en cualquier sector de la vida.

\section{Énfasis en Deporte (Networks 2)}

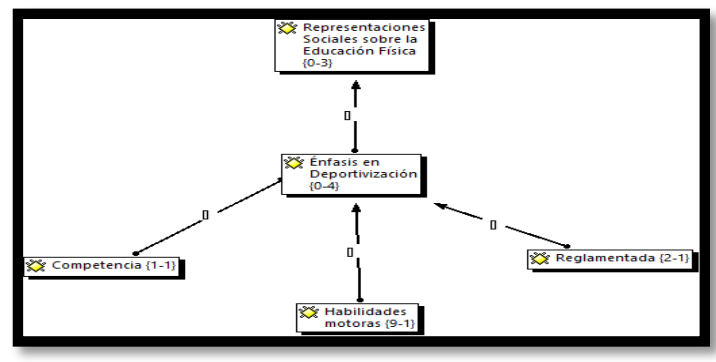

Figura 2. Énfasis en Deporte.

La competición debe ser entendida como medio de motivación y superación de uno mismo. La práctica deportiva tiene un conjunto de reglas que hay que respetar, porque no se puede practicar sin que exista un reglamento que permita cumplir un principio de igualdad entre todas las personas que están en dicha práctica. Las reglas no propician la desigualdad, sino que introducen un marco de actuación para todos y todas.

El deporte es uno de los medios que dispone la educación física para proporcionar al estudiante una formación integral. Como integrante del deporte no puede dejarse fuera del deporte educativo. Para que el deporte sea educativo debe incidir en el estudiante sobre tres aspectos fundamentales: aspectos psicomotrices, cognitivos y afectivos-sociales.

Debido a esto, no hay por qué introducir una práctica que tenga mayor representación social si con ello se alinea al sujeto a una práctica única. Esta selección significa que debe proponerse una educación en la que se origine un aprendizaje para disfrutar y disfrutar para aprender, donde exista una variedad de posibilidades que alcancen una mejora educativa en la práctica deportiva.

Práctica deportiva que no significa, en exclusiva, hacer deporte, entendido como un practicante más al uso, sino hacer deporte con todas las posibilidades que éste ofrece, y que no es sólo correr, saltar, lanzar, girar, etc., también significa que el alumno debe comprender las dificultades propias y de los demás, que existe múltiples posibilidades de participar: cómo me ayudan y cómo ayudo, cómo hago que los que saben menos puedan saber más, qué hago yo que los otros y otras no hacen o no pueden hacer, qué puedo hacer por ellos.

\section{Énfasis en Recreación (Networks 3)}

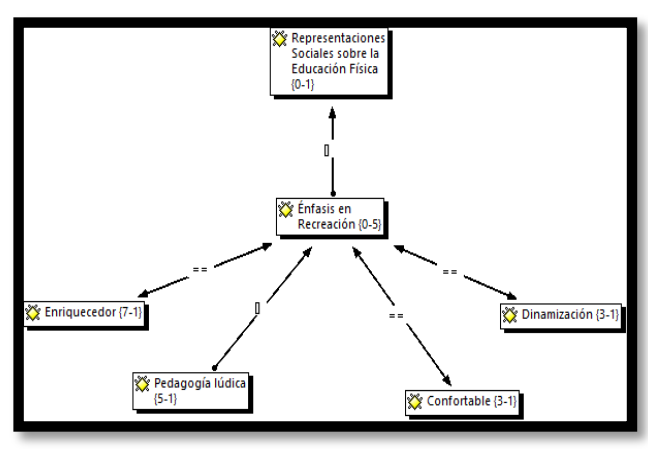

Figura 3. Énfasis en Recreación.

Probablemente, la diferencia entre las representaciones sociales de recreación pase más por la fuerza de cada uno de los cuatro elementos señalados en su relación. Entonces alguien enfatizará la libertad (es la actividad que se hace porque sí, porque la elegí); otro, el tiempo en que se da (es todo lo que hacemos cuando no nos obligan a hacer otra cosa); otro más priorizará lo placentero (es hacer lo que me gusta); etc. Podríamos seguir combinando posibilidades. Así aparecerán también los elementos más subjetivos diferenciados de los más objetivos. Aparecerán los juegos y sus teorías, etc. En general, no se utiliza la noción de recreación como sustantivo, como entidad de 
análisis, sino como adjetivo, como atributo de algo (la actividad). Tales formas del lenguaje suponen que "lo recreativo" es un carácter otorgado a un hacer concreto; y ese carácter tiene relación con el juego, con el tiempo libre, el entretenimiento, etc.

Por ejemplo, el recreo escolar justamente es una temporalidad que carece de importancia para los adultos pero que produce una gran felicidad a la mayoría de los usuarios en tanto se elimina "lo importante" para los docentes y aparece "lo importante" para los niños. Esa alegría del recreo, simbolizando aquí todo lo recreativo, ¿es tal porque no hay más obligación 0 por el recreo en sí mismo? Esta aparente oposición entre lo necesario y, generalmente, aburrido y monótono por un lado, y lo "libre" y placentero por el otro, ¿será la esencia de la recreación o sólo una contradicción en las concepciones del trabajo en esta sociedad?,. También, podemos pensar que ese recreo no es sino la contrapartida indisoluble o vuelta a un equilibrio perdido en la clase previa: esto es, cada recreo será diferente para los alumnos según la actividad anterior, la relación vincular con el docente, los permisos otorgados, etc.

¿Lo recreativo será entonces lo accesorio? ¿Será una compensación por el trabajo o por el cumplimiento de las obligaciones? ¿No podrá existir por sí mismo, con una identidad propia, caracterizada por lo creativo, libre, placentero, comprometido? Probablemente, si la dinámica de la clase fuese ágil; si los alumnos fueran protagonistas de la construcción del conocimiento; si las actividades fuesen placenteras e interesantes; si la participación de los educandos fuese intensa y comprometida los recreos no serían necesarios o la actual forma de estructurarlos no sería pertinente.

\section{Fase II Cuantitativa.}

El análisis que se aplicó a la encuesta fue el estudio de correspondencias que es un método de análisis de datos que representa gráficamente tablas de datos. El análisis de correspondencias es una generalización de una representación gráfica denominada diagrama de dispersión. Un diagrama de dispersión representa los datos en forma de puntos con relación a dos ejes de coordenadas perpendiculares: el eje horizontal, eje de las $x, y$ el eje vertical, eje de las $y$. Para el análisis de correspondencias, es conveniente reflexionar sobre lo que se entiende por diagrama de dispersión y sobre cómo se interpreta los datos que éste representa gráficamente.

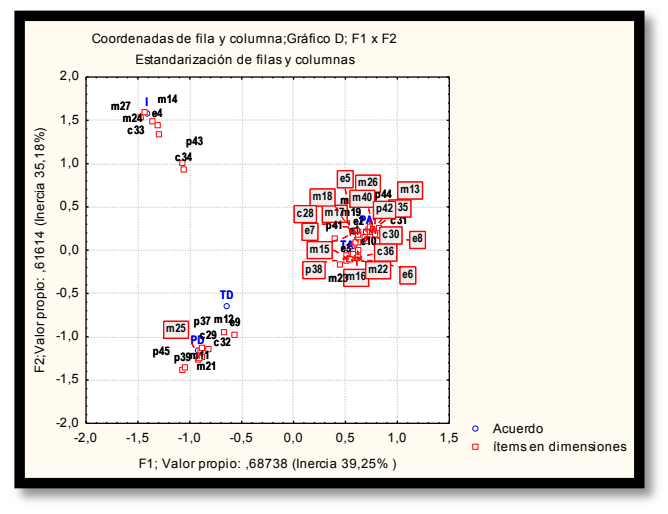

Gráfico 1. Coordenadas de fila y columna de los factores F1 y F2

El factor $\mathrm{F} 1$ tiene una inercia del $39,25 \%$ mientras que el segundo factor $F 2$, posee una inercia del $35,18 \%$. Cuando la inercia es baja, los perfiles fila presentan poca variación y se hallan cerca de su perfil medio, en tal caso se dice que existe poca asociación o correlación entre filas y columnas. Ambos factores, F1 y F2 suman una inercia de casi el $75 \%$ de la inercia, lo cual sugiere una alta asociación entre la escala y los ítems, por lo que puede usarse la segregación visual que se percibe de los ítems en el gráfico 1 en sus dimensiones del instrumento con las escala Likert (gráfico 1). 
La tabla que se muestra a continuación resume lo que se encuentra en el gráfico 1 , muestra los ítems y sus dimensiones que predominan en cada escala.

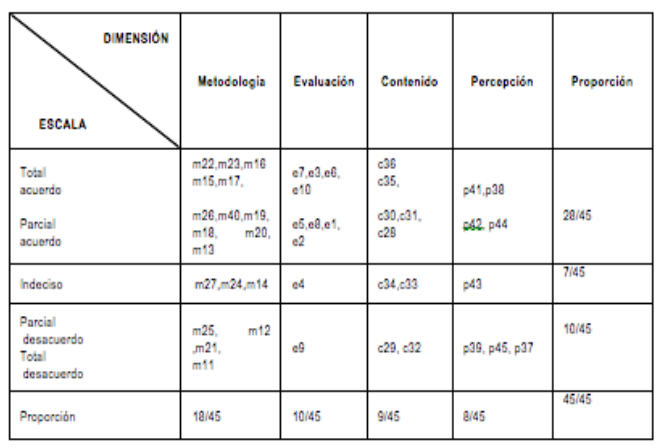

Tabla 3. Miembros similares según dimensiones, escala $e$ ítems del cuestionario. Factores 1 y 2.

La tabla 3, resume la información mostrada en el gráfico 1, los 45 ítems se distribuyeron solo en tres categorías de la escala original, pues se colapsan las categorías "total acuerdo" y "parcial acuerdo" en solo una, la categoría "indeciso" quedó aislada y del mismo modo las categorías "parcial desacuerdo" y "total desacuerdo" quedó colapsada en solo una. La mayoría de los ítems caen en la primera nueva categoría, la que apunta al acuerdo, el $62,22 \%$. El $22,22 \%$ de los ítems cae en las opciones que apuntan al desacuerdo, siendo la dimensión "percepción" la más desfavorable en términos del número de ítems involucrados, específicamente los ítems 37,39 y 45 . Las discusiones relevantes se enmarcan en los ítems que caen dentro de las opciones colapsadas al desacuerdo, por tal motivo, la separación visual que se percibe en el gráfico 1 y que se resumen en la tabla 1 para no descartar algún ítem es esencial para la construcción de las propuestas específicas anidadas en la propuesta clave de la actual investigación.

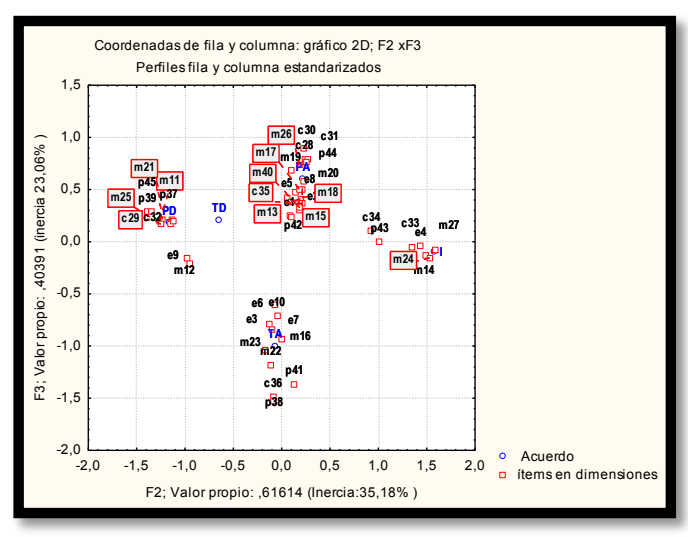

Gráfico 2. Coordenadas de fila y columna de los factores F2 y F3

El factor F2 tiene una inercia del 35,18\% mientras que el tercer factor posee una inercia del $23,06 \%$. Cuando la inercia es baja, los perfiles fila presentan poca variación y se hallan cerca de su perfil medio, en tal caso se dice que existe poca asociación o correlación entre filas y columnas. Ambos factores, F2 y F3 suman una inercia de casi el $60 \%$ de la inercia, lo cual sugiere una buena asociación entre la escala y los ítems, por lo que puede usarse la separación visual que se percibe de los ítems en sus dimensiones del instrumento con las escala Likert (grafico 2).

\begin{tabular}{|c|c|c|c|c|c|}
\hline ESCALA & METOOOLOCIA & EVALUACIÓN & CONTENDO & PERCEPCIÓN & PROPORCION \\
\hline $\begin{array}{l}\text { Tolal } \\
\text { acueroo }\end{array}$ & $\mathrm{m} 22 \mathrm{~m}, 23 \mathrm{~m}, \mathrm{~m} 6$ & $\begin{array}{c}87,83,86, \\
810\end{array}$ & 036 & p41,1038 & 1045 \\
\hline $\begin{array}{l}\text { Parcial } \\
\text { scuerco }\end{array}$ & 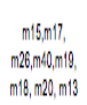 & $\begin{array}{c}05,08, \theta 1 \\
22\end{array}$ & $\begin{array}{c}035, \\
030,01,28 B\end{array}$ & DSh. P.4 & 18415 \\
\hline Irdociso & $\mathrm{m} 27, \mathrm{~m} 24, \mathrm{~m} 14$ & of & 034,33 & p13 & 745 \\
\hline $\begin{array}{l}\text { Parcial } \\
\text { desasuardo }\end{array}$ & $\begin{array}{c}\mathrm{m} 25, \mathrm{~m} 12, \mathrm{~m} 21, \\
\mathrm{~m} 11\end{array}$ & el & $C 28,62$ & p29, p15, p37 & 10415 \\
\hline $\begin{array}{l}\text { Tolal } \\
\text { dessouvero }\end{array}$ & . & . & . & . & 0445 \\
\hline Proporocoion & 1845 & 1045 & 945 & 845 & 4545 \\
\hline
\end{tabular}

Tabla 2. Miembros similares según dimensiones, escala e ítems del cuestionario. Factores F2 y F3. 


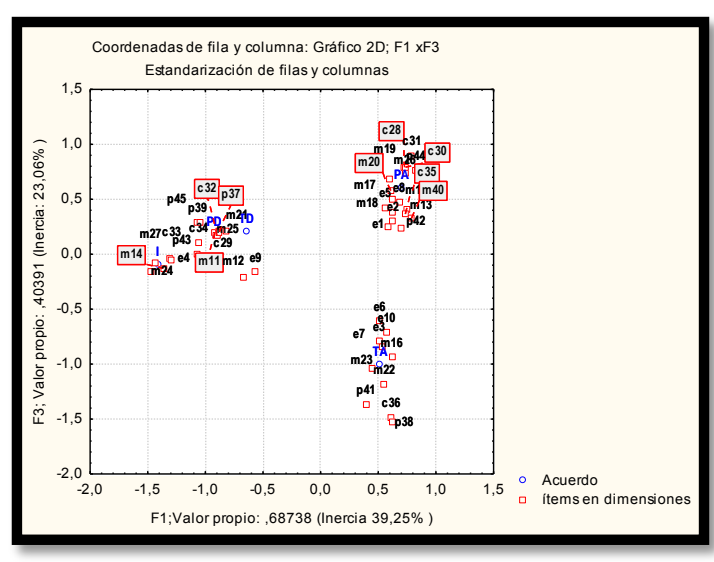

Gráfico 3. Coordenadas de fila y columna de los factores F1 y F3.

Como la mayoría de la información plasmada en la matriz original de los datos se resume en tres factores, solo resta evaluar el gráfico 3 , el cual muestra la inercia entre los factores F1 y F3. El factor $\mathrm{F} 1$ tiene una inercia del $39,25 \%$ mientras que el factor $\mathrm{F} 3$ posee una inercia del $23,06 \%$. Estos nuevos porcentajes evidencian una buena asociación o correlación entre filas y columnas, es decir, entre la escala Likert y los ítems de las dimensiones del cuestionario. Ambos factores, F1 y F3 suman una inercia de casi el $62 \%$ de la inercia, lo cual sugiere una buena asociación.

La tabla que se muestra a continuación resume lo que se encuentra en el gráfico 3, muestra los ítems y sus dimensiones que predominan en cada escala.

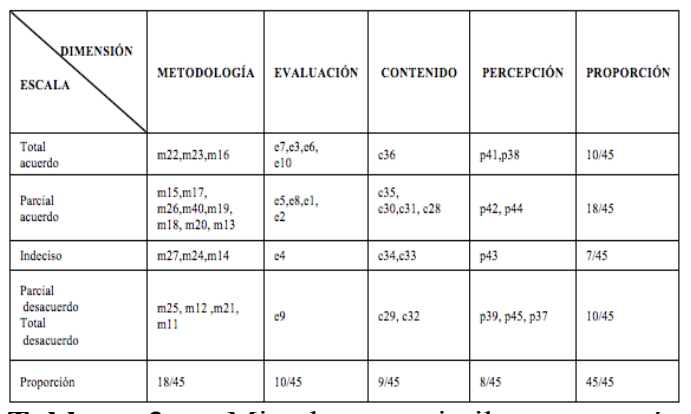

Tabla 3. Miembros similares según dimensiones, escala e ítems del cuestionario. Factores F1 y F3.

Desde la perspectiva de los factores F1 y F3 se percibe un agrupamiento similar, solo que ahora se notan unidas las opciones etiquetadas con "parcial desacuerdo" y "total desacuerdo". La distribución de los ítems en la tabla 3 es exactamente la misma a las tablas anteriores aunque aquí solo se agrupan las opciones al desacuerdo. Los ítems y dimensiones desfavorables nuevamente quedaron en las opciones que apuntan al desacuerdo y es en estas donde se extiende la discusión.

Para tener una mejor percepción, una tridimensional y no solo las proyecciones bidimensionales, se construyó el gráfico de los tres factores, los cuales poseen una inercia acumulada de alrededor del $98 \%$, es decir, solo tres componentes 0 factores explican la variabilidad de 45 ítems y 5 alternativas en la escala. Los gráficos 4 y 5 muestran el arreglo tridimensional, uno es solo una rotación del otro, sin embargo, aquí si se nota algo que casi casa desapercibido en los casos anteriores y el que la categoría etiquetada con "total desacuerdo" no aparece asociada a ningún ítem, lo cual se corresponde con los datos recolectados en campo. Esta perspectiva es la real ya aunque los gráficos anteriores y tablas anteriores muestran las agrupaciones finales de los ítems, no conviene asociar el "total desacuerdo" al "parcial desacuerdo", y los ítems que se colapsaron anteriormente en estas dos opciones realmente le pertenecen a la opción "parcial desacuerdo", la opción "total desacuerdo" no resultó relevante en la escala y por ende, los ítems y dimensiones desfavorables se asocian a la escala "parcial desacuerdo", lo que sugiere una separación de la realidad respondida no tan dramática, aun así, requerirá de la atención inmediata mediante la aplicación de medidas correctivas para no sesgar lo esperado en las respuestas con lo observado en campo. 


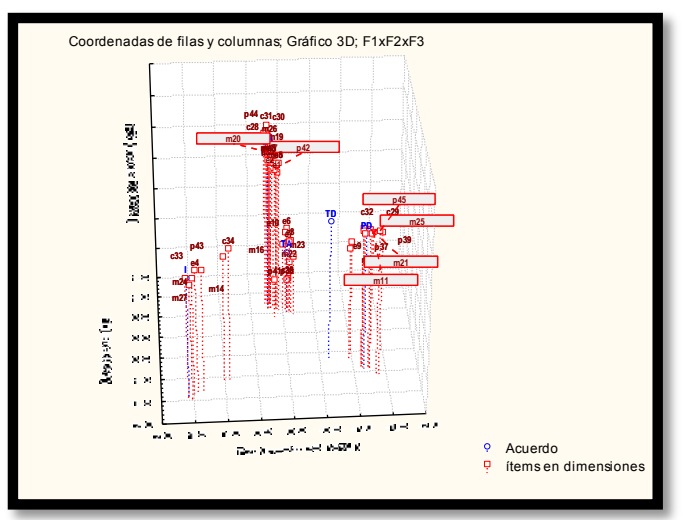

Gráfico 4. Coordenadas de fila y columna de los factores F1, F2 y F3 (Perspectiva 1)

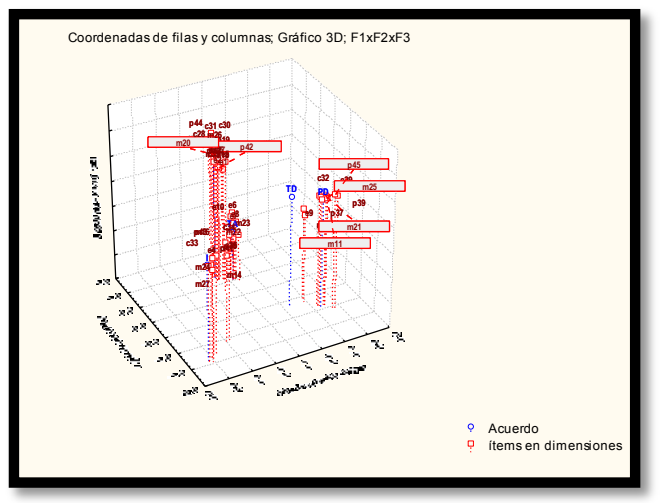

Gráfico 5. Coordenadas de fila y columna de los factores F1, F2 y F3 (Perspectiva 2).

En los gráficos anteriores aparece junto a la inercia, un valor propio, uno que aparecerá para cada factor involucrado.

En el gráfico 6 se muestran los valores propios, el valor de la distancia Chi-cuadrado, los grados de libertad asociados y el valor de probabilidad asociado a una prueba de independencia. Es evidente el rechazo de la hipótesis nula de independencia $(p<1 \%)$, por lo que definitivamente los ítems están asociados a la escala desde el punto de vista estadístico. Los valores propios para cada factor nos muestran cuanto puede explicar cada factor la variabilidad encontrada en los datos. Es notable como los tres primeros valores propios son los más altos y se corresponden con los tres factores utilizados para explicar la relación entre ítems y escala del cuestionario.

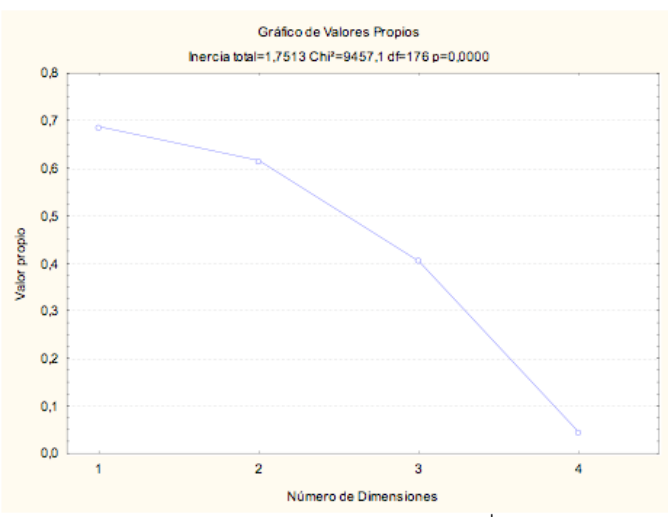

Gráfico 6 . Valores propios en los cuatro primeros factores

\begin{tabular}{|l|c|c|c|c|}
\hline & F1 & F2 & F3 & F4 \\
\hline TA & 0,210 & 0,004 & 0,787 & 0,000 \\
\hline PA & 0,539 & 0,053 & 0,408 & 0,001 \\
\hline I & 0,444 & 0,554 & 0,002 & 0,000 \\
\hline PD & 0,378 & 0,596 & 0,014 & 0,012 \\
\hline TD & 0,257 & 0,259 & 0,029 & 0,455 \\
\hline
\end{tabular}

Tabla 4. Cosenos cuadrados de los puntos-filas.

La tabla 4 muestra finalmente las opciones de la escala que más contribuyeron en cada factor. El primer factor y por ende sus ítems asociados se destacan por respuestas en el "parcial acuerdo", el segundo factor por las respuestas "indecisas" y "en desacuerdo" y el tercer factor por el "total acuerdo", por lo que el primer y tercer factor, es decir, F1 y F3 están en las mejores condiciones respecto a las respuestas dadas por los encuestados, por lo que sobre el segundo factor, F2, surgen las propuestas de la actual investigación, es decir, por los ítems que dominaron en este factor. 


\section{CONCLUSIONES}

La pedagogía como ciencia de la educación, en su función y encargo social de formación y mediación en el proceso socializador de los individuos, se vale de diferentes áreas de conocimiento e intervención práctica, que en su especificidad particular propenden por el desarrollo de un objetivo determinado. Entre ellas La Educación Física (en adelante EF), se presenta como campo disciplinar protagónico en las temáticas pedagógicas del cuerpo y del movimiento. Su concepción epistemológica, ha trasegado por diferentes significados acorde al paradigma filosófico de mundo, de ser humano y de su realidad existencial corpórea, dominante en un momento histórico determinado.

El movimiento es una de las cualidades innatas de los seres humanos de mayor importancia y relevancia, más allá del aspecto biológico y funcional. Es concebido como fuente de conocimiento, comunicación, sentimiento y emociones, salud, esparcimiento, placer, estética, desarrollo filogenético y ontogenético. Y como tal, merece ser revisado dentro del saber pedagógico y más exactamente sobre el conocimiento profesional de los profesores de educación física y deportes.

A través de la historia y de la evolución de los seres humanos, existen diversas disciplinas que han abordado el "estudio del movimiento" desde diferentes puntos de vista cientíicos y pedagógicos (fisiología, biomecánica, ergonomía, historia y sociología del deporte, ciencias médicas y educación física). La educación física que a partir de la reflexión pedagógica aborda procesos de interacción de diferentes disciplinas científicas como las ciencias biológicas y las ciencias sociales y humanas para solucionar campos de problemas mediante los siguientes objetos de estudio (el cuerpo y juego, el hombre en movimiento, educación del movimiento, cultura física, el movimiento como acción, cultura del movimiento, deporte, conducta motriz-acción motriz y experiencia corporal).

Los saberes mencionados anteriormente contribuyen a que los profesionales de la educación física aborden estructural y epistemológicamente el movimiento humano como contenido para mejorar su enseñanza en los espacios e instalaciones destinados a sus prácticas pedagógicas. Esto se debe a que el conocimiento del profesor está compuesto de un saber teórico y un saber práctico, siendo este último un "conjunto de conocimientos complejos, orientados a la practicidad que los profesores utilizan activamente para conformar y dirigir el trabajo de la enseñanza" (Elbaz,1983).

Desde esta perspectiva, los profesores no son sujetos pasivos y meramente ejecutores de postulados y teorías educativas elaboradas por expertos, son agentes que alcanzan un cierto grado de autonomía, que emplean en función de su experiencia profesional y de su singular modo de comprender e interpretar el contexto educativo en el que intervienen.

Este conocimiento práctico, a pesar de que para algunos autores que lo denominan el "conocimiento didáctico del contenido" está más focalizado en las teorías cognitivas de los docentes y máxime cuando se utilizan didácticas especificas en la enseñanza de actividades deportivas, recreativas y de motricidad en general que generan competencias en la condición física, habilidades técnicas, diversión y lúdica, entre otras.

La influencia de la corriente Físico-Deportiva, que desborda el campo curricular, ya que el deporte es considerado un fenómeno social que trasciende las intenciones mismas de su práctica representando factores antroposociológicos, políticos, económicos y educativos en el transcurrir de la historia de la humanidad. 
En este sentido los postulados se han fundamentado tradicionalmente en la concepción biologista y mecanicista del movimiento y sus características principales han sido la búsqueda del rendimiento, la representatividad en el deporte reglado.

Desde otra perspectiva, la corriente de La Educación Psicomotriz, como Cuerpo Pensante, aparece en el seno de la neurofisiología e incluso en la fenomenología; la cual emerge como oposición a la concepción exclusivamente biologista y mecanicista del cuerpo. El posicionamiento de esta corriente en el área de la educación y especialmente en la EF, se concibe como la educación dirigida a una entidad psicosomática que se resume básicamente en el "esquema corporal", en la cual las estructuras motrices se desarrollan en interacción constante entre el "yo" y el medio, ya sea físico o psicosocial.

Los aportes de esta corriente, en la multiplicidad de opciones que se abren a partir del descubrimiento de "todo un mundo de acción reeducativa y educativa a través del movimiento, aportando unos contenidos sin los cuales no se entendería la EF hoy".

La propuesta de Le Boulch "educación física funcional", se aleja del modelo tradicional de entrenamiento del cuerpo como máquina, y se acerca más a una perspectiva fenomenológica desde Buytendjik y de Merleau-ponty en la fenomenología de la ercepción. Esta propuesta trasciende la postura biológica y asume además los factores psicológicos ymsociales que condicionan el movimiento humano, fundamentándose especialmente en dos nociones "la del esquema corporal" y la del "esquema de acción.

La ciencia de la motricidad humana $(\mathrm{CMH})$, es un paradigma emergente planteado por el portugués Sérgio (1987), este autor sustenta un estatuto ontológico en la ciencia de la motricidad humana $(\mathrm{CMH})$, en tanto busca figurar en el proceso histórico del conocimiento, partiendo de la $E F$, en sus fundamentos cartesianos y empiristas, hasta posicionarse como ciencia: $\mathrm{CMH}$, donde la fenomenología y la hermenéutica predominan reconociendo la primacía de lo intencional, y del sentido del movimiento humano, sobre lo meramente físico. Su propuesta central, como puede leerse en la mayoría de sus publicaciones, se presenta como una crítica ininterrumpida contra todos los dualismos tradicionales: empirismoracionalismo, cuerpo-alma, hombre-mundo; incitando a la superación de lo físico del ser y del logos hacia la dialéctica del acto y de la relación.

Es un planteamiento que en expresión del autor, emerge y tiene su historia en la $\mathrm{EF}$, pero la trasciende; no pretende reemplazarla, sino surgir como una ciencia, con todo lo que para ello se requiere -identidad propia, objeto de estudio, corpus teórico-, invitando a la participación de todas aquellas áreas y disciplinas que se proyecten hacia lo humano; en tanto que la motricidad entendida como una de las dimensiones centrales del ser y potencialidad para su desarrollo integral, no es un asunto a ser tratado desde una sola área del conocimiento.

\section{REFERENCIAS BIBLIOGRÁFICAS}

Ander-Egg, E. (2000). Cómo organizar el trabajo de investigación. Métodos y técnicas de investigación III.

Azorín, F. Y., \& Sánchez-Crespo, R. JL 1986. Métodos y Aplicaciones del Muestreo. Alianza Universidad. Madrid.

Balcells, M. C., Foguet, O. C., \& Argilaga, M. T. A. (2013). Métodos mixtos en la investigación de las ciencias de la actividad física y el deporte/Mixed Methods in the Research of Sciences of Physical Activity and Sport. Apunts. Educació física i esports, (112), 31.

Creswell, J. W., Plano Clark, V. L., Gutmann, M. L., \& Hanson, W. E. (2003). Advanced mixed methods research designs. Handbook of mixed 
methods in social and behavioral research, 209240.

Creswell, J. W., \& Clark, V. L. P. (2007). Designing and conducting mixed methods research.

Creswell, J. W. (2009). Editorial: Mapping the field of mixed methods research.Journal of Mixed Methods Research, 3(2), 95-108.

Creswell, J. W., Klassen, A. C., Plano Clark, V. L., \& Smith, K. C. (2011). Best practices for mixed methods research in the health sciences. Bethesda, MD: National Institutes of Health, 10.

D'andrea, A. M., \& Corral de Zurita, N. (2006). Representaciones sociales de formadores de formadores sobre el éxito y el fracaso académico y el buen y el mal estudiante. Actas de la XIII Reunión de Comunicaciones Científicas y Tecnológicas. CorrientesArgentina. Recuperado de: http://www. unne. edu. ar/Web/cyt/cyt2006/index. htm.

Elbaz, F. (1983). Teacher Thinking. A Study of Practical Knowledge. Croom Helm Curriculum Policy and Research Series. Nichols Publishing Company, 155 West 72nd Street, New York, NY 10023.

Glaser, B. G. A. L. (86). Strauss (1967): The Discovery of Grounded Theory: Strategies for Qualitative Research. London: Wiedenfeld and Nicholson, 81.

Greene, J. C., \& Caracelli, V. J. (2003). Making paradigmatic sense of mixed methods practice. Handbook of mixed methods in social and behavioral research, 91-110.

Hernández, J. G., Herrera, L., Martínez, R., Páez, J. G., \& Páez, M. A. (2011). Teoría fundamentada. Seminario: Generación de Teoría. Facultad de Humanidades y Educación, Universidad del Zulia.
Hesse-Biber, S. N. (2010). Mixed methods research: Merging theory with practice. Guilford Press.

Johnson, B., \& Turner, L. A. (2003). Data collection strategies in mixed methods research. Handbook of mixed methods in social and behavioral research, 297-319.

Krippendorff, K., \& Krippendorff, K. (1990). Metodología de análisis de contenidoteoría y práctica (No. 001.42 K71).

Mora, M. (2002). La teoría de las representaciones sociales de Serge Moscovici. Athenea digital: revista de pensamiento e investigación social, (2), 078102.

Robert, S. (1994). Investigación con estudio de casos. cap, 1, 15-21.

Sandín Esteban, M. P., \& Esteban, M. P. S. (2003). Investigación cualitativa en educación: fundamentos y tradiciones.

Selltiz, C. (1980). Métodos de investigación en las relaciones sociales. Ediciones Rialp.

Strauss, A. L., \& Corbin, J. (2002). Bases de la investigación cualitativa: técnicas y procedimientos para desarrollar la teoría fundamentada. Medellín: Universidad de Antioquia.

Tashakkori, A., \& Teddlie, C. (2010). Putting the human back in "human research methodology": The researcher in mixed methods research. Journal of Mixed Methods Research, 4(4), 271-277.

Teddlie, C., \& Tashakkori, A. (2003). Major issues and controveries inthe use of mixed methods in the social and behvioral sciences. Handbook of mixed methods in social $\&$ behavioral research,3-50. 